\title{
Gadolinium/terbium hybrid macromolecular complexes for bimodal imaging of atherothrombosis
}

Nguyễn Trọng Nghĩa

Eric Tinet

Dominique Ettori

Anne Beilvert

Graciela Pavon-Djavid

Murielle Maire

Phalla $\mathrm{Ou}$

Jean-Michel Tualle

Frédéric Chaubet 


\title{
Gadolinium/terbium hybrid macromolecular complexes for bimodal imaging of atherothrombosis
}

\author{
Nguyễn Trọng Nghĩa, ${ }^{a, b}$ Eric Tinet, ${ }^{a}$ Dominique Ettori, ${ }^{a}$ Anne Beilvert, ${ }^{b}$ Graciela Pavon-Djavid, ${ }^{b}$ Murielle Maire, ${ }^{b}$ \\ Phalla Ou, ${ }^{c, d}$ Jean-Michel Tualle, ${ }^{a, t}$ and Frédéric Chaubet ${ }^{b, t, *}$ \\ aUniversity Paris 13, Institut Galilée, University Sorbonne Paris-Cité, Laser Physics Laboratory, CNRS UMR 7538, Villetaneuse, France \\ bUniversity Paris 13, Institut Galilée, University Sorbonne Paris-Cité, Laboratory for Vascular Translational Science, INSERM U1148, Villetaneuse, \\ France \\ 'University Paris-Diderot, CHU X. Bichat, University Sorbonne Paris-Cité, Laboratory for Vascular Translational Science, INSERM U1148, Paris, \\ France \\ ${ }^{d}$ Hôpital Bichat, AP-HP, Paris, France
}

\begin{abstract}
We developed a fluorescence imaging microscope system intended for the localization within artery slices of a gadolinium-based macromolecular biospecific magnetic resonance (MR) contrast agent used for the visualization of atherothrombosis. As the contrast agent is not initially fluorescent, we substitute some gadolinium ions for terbium ions to make them fluorescent while preserving their chemical characteristics. A long fluorescence emission time constant enables us to have a suitable signal-to-noise ratio, despite a low intensity, using pulsed illumination and time-gated imaging. Images of rat arteries show that the contrast agent is indeed localized on the specific regions of the tissues. We currently have a tool that allows us to understand and optimize the MR contrast agent. @ 2017 Society of Photo-Optical Instrumentation Engineers (SPIE) [DOI: 10.1117/1.JBO.22.7.076004]
\end{abstract}

Keywords: optics; fluorescence microscopy; macromolecular contrast agents; molecular imaging; MRI; atherosclerosis.

Paper 170054R received Jan. 24, 2017; accepted for publication Jun. 15, 2017; published online Jul. 11, 2017.

\section{Introduction}

Cardiovascular diseases (CVD) are the leading cause of death in the world. ${ }^{1}$ The main expression of CVD is atherosclerosis, a complex pathological phenomenon leading to the formation of a rigid plaque at the inner surface of the vascular wall. ${ }^{2}$ Acute coronary syndrome and stroke are the main consequences of thrombus formation, namely atherothrombosis, that occurs either following a massive plaque rupture or from intraplaque hemorrhage. ${ }^{3}$ Medical imaging is the most widespread and efficient tool for the diagnosis of atherothrombosis, and molecular imaging is a recent and promising development in medical imaging that is dedicated to the visualization of the biological processes at the molecular and cellular levels. ${ }^{4}$ Various molecular imaging modalities have been used for the diagnosis of atherosclerosis. The commonly used imaging modalities include computed tomography (CT), positron emission tomography (PET), single photon emission computed tomography (SPECT), magnetic resonance imaging (MRI), contrast-enhanced ultrasound, optical bioluminescence, and optical fluorescence; each imaging modality has its own advantages and limitations. ${ }^{5,6}$ PET and SPECT are highly sensitive and quantitative with a relatively poor spatial resolution, MRI provides high-spatial resolution images with excellent soft tissue contrast but suffers from low sensitivity, and optical imaging techniques offer a spatial resolution superior to a conventional clinical imaging platform but with limited penetration depth. ${ }^{7}$ However, real-time endoscopecompatible microscopic systems, such as multiphoton laser scanning microscopy ${ }^{8}$ optical coherence tomography, ${ }^{9}$ and photoaccoustic tomography, ${ }^{10}$ are available to allow sensitive observing and questioning of cellular and molecular functions

*Address all correspondence to: Frédéric Chaubet, E-mail: frederic.chaubet@ univ-paris13.fr

${ }^{\dagger}$ Equal contribution to the study. in living subjects. Combinations of imaging techniques are designed to take advantage the strengths of modalities while minimizing their limitations, and hybrid systems that combine two or more imaging modalities, such as PET/CT, SPECT/CT, and PET/MRI, are commercially available. ${ }^{4}$

MRI coupled with intravenous injection of low-molecular weight gadolinium $(\mathrm{Gd})$-based contrast agents allows the evaluation of the degree of artery stenosis (angiography) due to the development of atherosclerotic plaques. Because of their small size, these complexes diffuse rapidly outside the vascular space and are quickly excreted through the kidneys. ${ }^{11}$ MRI is also a gold standard in clinical use ${ }^{12}$ for detecting intraplaque hemorrhages in at-risk atherothrombotic patients. ${ }^{13}$ Using dedicated magnetic resonance (MR) sequences, MRI is also able to provide information about plaque components such as lipid-rich necrotic core, intraplaque hemorrhage, calcification, and surface defects including erosion, disruption, and ulceration. ${ }^{14}$ However, because the best MRI resolution are a few dozen micrometers, ${ }^{15}$ long acquisition times are required to get useful images. Thus, this limitation is not suitable for the study of the distribution of the MRI agents in small volumes like in a single cell or even into the layers of a small artery wall. ${ }^{15,16}$ Yet, if we want to study why a given agent exhibits some affinity for a pathological tissue or if we want to modify the agent to increase this affinity, we need a very high-resolution imaging method such as optical microscopy.

The issue is therefore to develop a bimodal contrast agent, allowing a contrast for both MRI and optical microscopy. Many efforts to synthesize fluorescent MRI agents have been made. ${ }^{17}$ However, biological tissues are naturally fluorescent, too, although not intensely fluorescent, but modified MRI agents are not very bright either. To make things worse, emission wavelengths of the main fluorophores used for agents fall in the

$1083-3668 / 2017 / \$ 25.00$ @ 2017 SPIE 
emission regions of the tissues autofluorescence, making it sometimes difficult to interpret the images. Hence, we need to develop more sophisticated markers, for example, fluorophores capable of emitting in the near-infrared where the fluorescence of the tissues is absent. ${ }^{18}$ Another way to solve this issue is to use fluorescent emitters that decay very slowly, a lot longer than the time constant of the autofluorescence decay. Lanthanides possess specific magnetic and electronic properties; in particular ions of terbium and europium, which belong to the middle of the lanthanide series, exhibit unique fluorescence properties that have been widely exploited for biological and biochemical assays. ${ }^{19-23}$ They have several advantages compared with purely organic fluorophores: ${ }^{24,25}$ very long luminescence decay (some hundred microseconds to some milliseconds), better photostability, narrow emission lines, and large Stokes shift. These compounds can therefore achieve fluorescence optical imaging with a time-resolved emission in the visible spectrum (green light for terbium and red light for europium) easily discriminated from the background noise, with detection sensitivities down to femtomolar. ${ }^{26}$ Moreover, in addition to characteristic electronic configuration, lanthanides exhibit a chemical resemblance with close atomic and covalent radii with the most stable oxidation state of $+3 .^{27}$ An MR contrast agent that would have its $\mathrm{Gd}^{3+}$ ions replaced by $\mathrm{Tb}^{3+}$ ions cannot be noticeably different chemically from the pure MR one, and it is expected to show similar biodistribution and/or cellular uptake profiles so that combined MRI and optical imaging protocols become possible. ${ }^{28}$ Therefore, any useful information collected using the modified agents remains pertinent for the original agent.

For this study, we used a chelating macromolecule, named P717, made of dextran substituted with carboxylate and GdDOTA groups ${ }^{29}$ (see Fig. 2). This long circulating blood pool contrast agent is devoid of biospecificity for arterial walls. ${ }^{30}$ In previous works, P717 substituted with sulfate groups was able to bind to human-activated platelets and allowed imaging of atherosclerosis plaque in a mouse model. ${ }^{31,32}$ Then, P717 substituted with trityrosine residues was evidenced as an MR molecular imaging agent able to bind in vivo to lipoproteins used as transporters of cholesterol and other lipids ${ }^{33}$ within the atherosclerotic plaque. Both studies raised the possibility of using modified polysaccharides as targeting moieties for the imaging and/or treatment of vascular diseases.

The main objective of the study was to obtain usable images of diseased vascular tissues using an optical imaging modality in which sensitivity and resolution are complementary to that of MRI. For that purpose, we prepared P717 by replacing Gd ions with terbium ions. The chelate limits the quenching of the luminescence from direct interaction of the water molecules with the ions and eventually allows the link to a biomolecule for targeting purposes. To overcome the weak absorption cross section of europium and terbium ions, chelate complexes should contain an organic chromophore acting as an antenna, absorbing excitation light and transferring energy to the lanthanide. ${ }^{21}$ However, such amplifier antennas are aromatic structures poorly soluble in physiological media, and avoiding their use would be beneficial, reducing both costs and potential side toxicity of the complexes. As the quantum yields and fluorescence lifetimes are better for terbium ions than for europium ions, ${ }^{26,34-36}$ we have developed an optical system to visualize $\mathrm{Tb}$ luminescence in biological samples without the use of an antenna. The excitation wavelength in near UV for $\mathrm{Tb}$ (in the visible domain for
$\mathrm{Eu}$ ) is one of the challenges that have been addressed. Eventually, the biospecificity of the P717 complexes was achieved by conjugation with a low-molecular-weight fucoidan that was established as a relevant targeting agent for molecular imaging of atherothrombosis. ${ }^{37,38}$ Animal experiments have been performed with a rat model of aneurysmal thrombosis. ${ }^{38}$

\section{Time-Gated Imaging System}

The scheme of the time-gated imaging system is shown in Fig. 1. It is based on a Zeiss inverted microscope model Observer Am1. The UV excitation source is a laser diode Stradus 375 from Vortran Medical Technology, whose emission wavelength peaks at $371 \mathrm{~nm}$. Its maximum power in continuous wave mode is $15 \mathrm{~mW}$. The laser beam is collimated by the buildin optics system. In pulse mode, a very weak parasitic UV light is still emitted some milliseconds after turn off. It causes autofluorescence noise of the artery wall that may exceed the fluorescence signal of terbium ions accumulated in the artery slice. We added the chopper C from EG\&G Instruments (model 651) to eliminate this noise. A filter F1 eliminates the parasitic visible light from the excitation laser, and a second filter F2 is placed after the microscope objective to block the UV laser light $(371 \mathrm{~nm})$ from propagating further into the microscope and possibly creating autofluorescence. To avoid parasitic light accumulation, a shutter protects the camera and is only opened during the image acquisition. For this purpose, we used a hard disk voice-coil actuator with a razor blade instead of the read/write heads. The whole acquisition process is synchronized using a PIC18F4550 microcontroller.

The laser light is focused on the sample by a convergent lens with a 10-mm focal length, and the laser spot is scanned on the sample to get the whole fluorescent image: we take a fluorescence image for each position of the spot, and these data are then merged into a single fluorescence image. This simple setup provides a high enough excitation light density. This density can be adjusted by defocusing the lens away from the sample: the size of the laser spot changes accordingly. The maximum illumination density corresponds to the smallest diameter of the laser spot, which is $8 \mu \mathrm{m}$.

The camera is an Andor iXon3 DU-897D EMCCD camera. We can record white light images using a halogen lamp $\mathrm{H}$ (eventually colored through the use of a filter set FS corresponding to the primary colors) or time-gated fluorescence images using the procedure described above. We also have the possibility of inserting a spectrometer: as the position of the laser light is

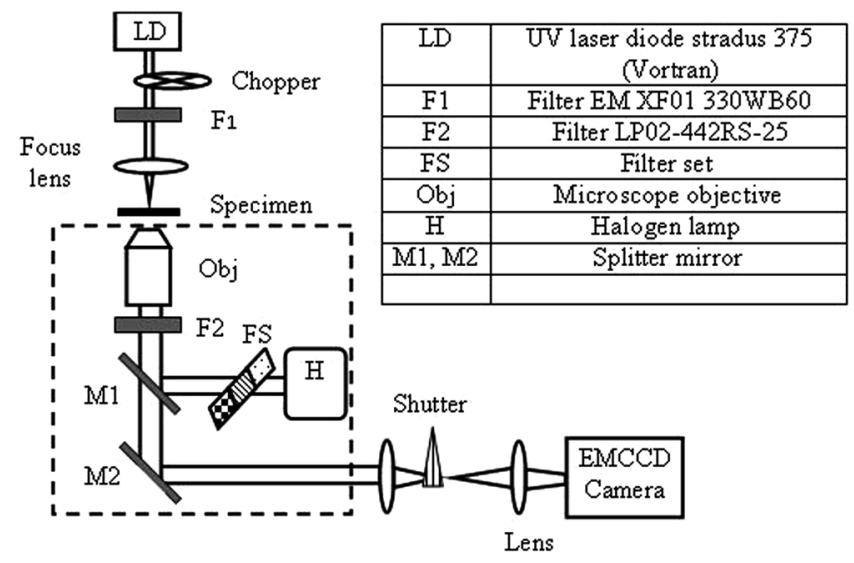

Fig. 1 Schematics of the time-gated fluorescence microscope. 
well controlled, we can thus obtain the fluorescence spectrum of any point in the recorded image, which will ensure the origin of the collected fluorescence.

\section{Materials and Methods}

All chemicals, purchased from Sigma-Aldrich (St Quentin Fallavier, France), were of reagent grade, and deionized water was used. The starting material, named LP717 in the paper, was kindly provided by Guerbet Company. LP717 is a carboxymethylated dextran substituted with a chelating moiety $1,4,7$, 10-tetraazacyclododecane-1,4,7,10-tetraacetic acid (DOTA) via a diaminoethyl linker. The average degrees of substitution (DS) in the different chemical groups are: DS(carboxymethyl) $=$ $0.32 \pm 0.02(1.14 \pm 0.05$ meq COO- $/ \mathrm{g})$ and DS(DOTA $)=$ $0.23(\% N=4.43 \pm 0.30 \% \mathrm{w} / \mathrm{w})$. The molecular weights are $\overline{\mathrm{Mn}}=29,000 \mathrm{~g} / \mathrm{mol}$ (average number weight) and $\overline{\mathrm{Mw}}=$ $49,000 \mathrm{~g} / \mathrm{mol}$ (average weight weight) with a hydrodynamic radius $(\mathrm{Rh})$ of $6.0 \pm 0.3 \mathrm{~nm} .{ }^{33}$ Low-molecular-weight fucoidan from the brown seaweed Ascophyllum nodosum was purchased from Algues et Mer (Ile d'Ouessant, France). Chemical composition of the starting fucoidan $(\mathrm{w} / \mathrm{w})$ is: fucose, $44.7 \pm 5.4 \%$; uronic acids, $22.8 \pm 5 \%$; and sulfate groups, $17.6 \pm 2.5 \%$. $\overline{\mathrm{Mn}}=5000 \mathrm{~g} / \mathrm{mol} ; \overline{\mathrm{Mw}}=10,000 \mathrm{~g} / \mathrm{mol}$. Fucoidan was aminated with diaminopropane at its reducing end as previously described. ${ }^{38}$ Chemical composition of aminated fucoidan (w/w) is: fucose, $39.4 \pm 5.0 \%$; uronic acids, $13.8 \pm 1.5 \%$; and sulfate groups, $29.7 \pm 2.0 \%$ with $\overline{\mathrm{Mn}}=7800 \mathrm{~g} / \mathrm{mol}$ and $\overline{\mathrm{Mw}}=10,600 \mathrm{~g} / \mathrm{mol}$. The amount of primary amine, determined with phthalaldehyde colorimetric assay using bromopropylamine as the standard, ${ }^{39}$ is $0.89 \pm 0.08$ ( $\mathrm{mol} / \mathrm{mol}$ of fucoidan).

\subsection{Preparation of P717 Metal Complexes (P717M)}

The chelation of $\mathrm{LP} 717$ by $\mathrm{Gd}^{3+}$ or $\mathrm{Tb}^{3+}$ ions was performed by mixing $\mathrm{GdCl}_{3}, 7 \mathrm{H}_{2} \mathrm{O}$ or $\mathrm{TbCl}_{3}, 7 \mathrm{H}_{2} \mathrm{O}(1 \mathrm{Gd}$ or Tb per DOTA groups) with LP717 in water. After adjusting the $\mathrm{pH}$ to 6.5 with $\mathrm{NaOH}$, the solution was maintained at room temperature overnight followed by heating at $60^{\circ} \mathrm{C}$ for $4 \mathrm{~h}$. Chlorides were removed by dialysis and white products were obtained by freeze-drying. The products named P717Gd and P717Tb underwent the same coupling to aminated fucoidan. The total $\mathrm{Gd}$ and $\mathrm{Tb}$ contents were determined with inductively coupled plasma atomic emission spectroscopy (ICP-AES) by Service de Microanalyse du CNRS (Vernaison, France).

\subsection{Coupling of P717M with Fucoidan}

The P717M ( $\mathrm{M}=\mathrm{Gd}$ or $\mathrm{Tb}$ ) was then linked to aminated fucoidan by a coupling reaction with conventional agents EDC/NHS [1-ethyl-3-(3-dimethylaminopropyl) carbodiimide hydrochloride/ $N$-hydroxysuccinimide]. Briefly, $300 \mathrm{mg}$ of P717M were dissolved in $5 \mathrm{ml}$ of bidistilled water. $20 \mathrm{mg}$ of EDC and $12 \mathrm{mg}$ of NHS were added, and stirring was maintained for $15 \mathrm{~min}$ at room temperature. $200 \mathrm{mg}$ of aminated fucoidan were added to the mixture ([fucoidan $]=40 \mathrm{mg} / \mathrm{ml}$ ) and maintained under agitation for $2 \mathrm{~h}$. Purification was performed by dialyzing the suspension against $\mathrm{NaCl} 1 \mathrm{M}(2 \times)$ and bisdistilled water $(5 \times)$ (MWCO 12,000). Finally, the conjugate was obtained as a fluffy powder after freeze-drying in about $60 \%$ yield. The fucoidan content was obtained by a sulfate content colorimetric assay as previously described. ${ }^{37}$

\subsection{Determination of the Molecular Weights}

Molecular weights were determined by size-exclusion chromatography (SEC) on a TSK gel G3000 PWXL (Interchim SA, France) with a LC10Ai pump (Shimadzu, France) and phosphate-buffered saline (PBS), and $\mathrm{LiNO}_{3} 150 \mathrm{mM} \mathrm{pH}=7.4$ was the eluent at a flow rate of $0.5 \mathrm{ml} / \mathrm{min}$ at $25^{\circ} \mathrm{C}$. Determination was performed with a spectrophotometric detector multiangle laser light scattering (MALLS) (Dawn EOS, Wyatt Technology Corp., Santa Barabara, California) and a differential refractometer RID 10 A (Shimadzu, France). Data have been exploited with the Astra 5.3.4.14 software (Zimm order 1 with angles between $52 \mathrm{deg}$ and $142 \mathrm{deg}$ ).

\subsection{Relaxivity Study}

Dilutions of P717Gd and P717Gd-F were prepared in PBS (10, $5,2,1,0.5$, and $0.1 \mathrm{mg} / \mathrm{ml}$ ) and distributed in tubes. The longitudinal relaxation times $\mathrm{T} 1$ were measured with the small animal 7T MRI. The relaxation rate $(1 / T 1)$ was plotted against Gd concentration values, and longitudinal relaxivity $(r 1)$ was calculated from the slope of the graph.

\subsection{Animal Study}

We performed investigations on rat abdominal aortas. The procedure and the animal care complied with the "Principles of animal care" formulated by the EU (Animal Facility Agreement 75-18-03; 2005), and animal experimentation was performed under the authorization 75-101 of the French Ministry of Agriculture after approval by the University Ethical Committee.

\subsubsection{Induction of the aneurysmal thrombus}

Under intraperitoneal pentobarbital anesthesia $(4 \mathrm{mg} / 100 \mathrm{~g}$ bodyweight; Ceva Santé Animale, Paris, France), 20 mm of the infrarenal aorta (beginning $2 \mathrm{~mm}$ below the left renal artery) was separated from the vena cava. Collateral arteries were dissected from surrounding connective tissue, ligated in two places, and cut between them. The aorta was clamped and four units of porcine pancreatic elastase (E-1250; Sigma-Aldrich) in $550 \mu \mathrm{l}$ $\mathrm{NaCl} 9 \%$ was perfused transmurally during $1 \mathrm{~h}$, using an automatic pressure perfusion pump. The segment was then rinsed, the catheter removed, the entry hole closed by suture, and flow re-established. ${ }^{40}$ A total of 12 male 7-week-old Wistar rats from CERJ (Le Genest, France) were prepared. To localize the treated segment during the MRI session, the distances between the upper and lower points of the perfused segment and the origin of the left renal artery were measured with the microscope eyepiece, and surgical wounds were closed. This model is characterized by the presence of an intraluminal thrombus 1 to 3 weeks after aneurysm induction. ${ }^{40}$ To increase the size and the activity of the aneurysmal thrombus, injections of Porphyromonas gingivalis were performed at day 8 and day 16 . Four rats died before the MRI sessions.

\subsubsection{MRI session}

Rats were anesthetized with isofluorane before injection of the macromolecular agents in the vein of the tail. They were scanned with a 7T small animal MRI (Bruker, Germany) before and after injection using a dedicated coil. For black-blood highresolution imaging of the aortic vessel wall, a RARE T1 sequence with cardiac gating was used with the following 

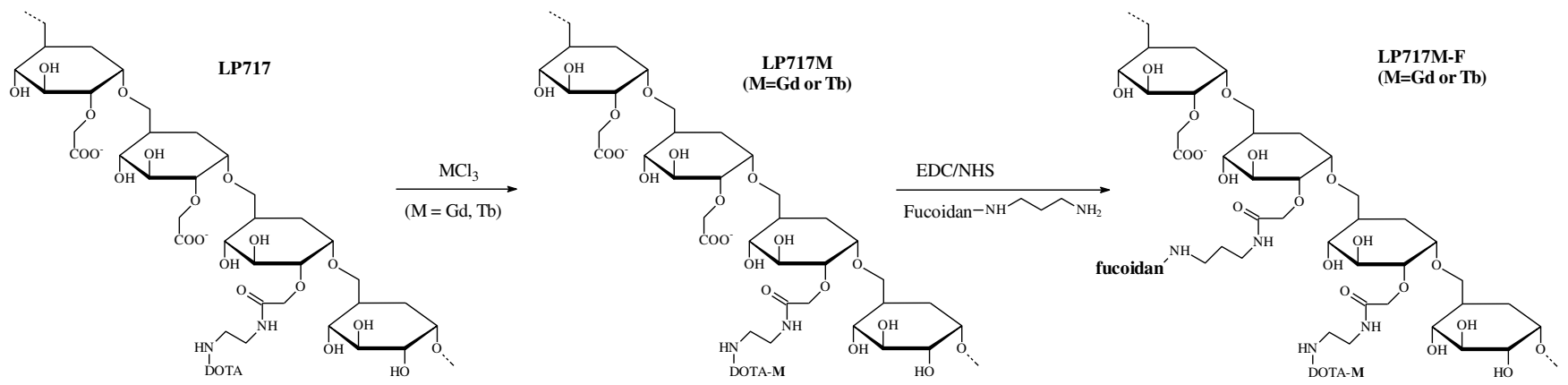

Fig. 2 Schematic structures and synthesis of P717M-F (with $M=G d$ or $T b$ and $F=$ fucoidan).

parameters: minimum $\mathrm{TR}=1741.9 \mathrm{~ms}, \mathrm{TE}=9 \mathrm{~ms}, \mathrm{FOV}=$ $5 \mathrm{~cm}$, and a matrix size of $256 \times 256$. Continuous slices (slice thickness $=0.3 \mathrm{~mm}$ ) were made under the renal aorta bifurcation. Image analysis was performed using OsiriX software (DICOM reader v3.7). Maximum luminal narrowing was quantified by the percentage reduction $(\Delta R \%)$ of aortic luminal area as previously published. ${ }^{41}$ Arterial wall contrast agent uptake analysis was performed on slices that corresponded to histology sections. Intraluminal areas that evidenced visual intraluminal hypersignal from $10 \mathrm{~min}$ to $1 \mathrm{~h}$ after injection of contrast agent were manually contoured for quantitative signal analysis. Regions of interest were pasted on all corresponding MR images. The signal-to-noise ratio (SNR) of aortic wall thrombus was measured by calculating the average signal intensity in the region of interest from MR images at each imaging point $[\mathrm{SNR}=($ SIaortic wall - SImuscle $) /$ SDnoise signal $]$. Normalized signal enhancement $[\Delta \mathrm{NSE} \%=(\mathrm{SNR} t-\mathrm{SNR}$ before $) /$ SNRbefore $\times 100]$ was calculated at 10,20 , and 30 min after injection and analyses were performed between each scan time. ${ }^{42}$ At the end of the session, animals were euthanized and the perfused portion of the aorta, including the dilatation, was harvested and flushed with PBS. Then the aorta was cut transversally into 2-mm-width tissue rings, which were frozen at $-20^{\circ} \mathrm{C}$ in cold isopentane. Healthy artery parts were used as standard samples.

\subsection{Fluorescence Microscopy Study}

\subsubsection{Pathological artery}

Frozen artery rings were cut into $6-\mu \mathrm{m}$ sections and placed onto a quartz lamella. For fluorescence images recording, we used the $20 \times$ magnification objective. The laser's spot diameter is $56 \mu \mathrm{m}$. Laser power is $5 \mathrm{~mW}$ in $\mathrm{CW}$ mode. Therefore, the average intensity of laser on the specimens is $250 \mathrm{~W} / \mathrm{cm}^{2}$. During the experiment, the laser emits a squared pulse of light with a repetition rate of $7.3 \mathrm{~Hz}$ and a pulse duration of $6.2 \mathrm{~ms}$.

\subsubsection{Ex vivo incubation of P717Tb with healthy artery}

The rat healthy artery is placed in PBS for $24 \mathrm{~h}$ at $37^{\circ} \mathrm{C}$ under gentle shaking with P717Tb $\left[10 \mathrm{mg} / \mathrm{ml},\left(\mathrm{Tb}^{3+}\right)=\right.$ $4.6 \times 10^{-3} \mathrm{~mol} / \mathrm{l}$. A standard sample is prepared without $\mathrm{P} 717 \mathrm{~Tb}$. Then, the artery is frozen in cold isopentane before being treated as the pathological artery.

\section{Results and Discussion}

\subsection{Bimodal Contrast Agents}

P717Gd and P717Tb were obtained by treatment of LP717 with $\mathrm{GdCl}_{3}$ and $\mathrm{TbCl}_{3}$, respectively, before coupling with aminated fucoidan (Fig. 2). Table 1 shows the main physicochemical characterizations. The amount of fucoidan was estimated by the sulfate assay (see Sec. 3). As expected, data are independent from the cation used.

Absolute molecular weights have been obtained from MALLS coupled with differential refractometry. By taking into account the degree of substitution of the different substituting

Table 1 Physicochemical characteristics of LP717 and P717 complexes.

\begin{tabular}{lcccccc} 
Name & $\begin{array}{c}\overline{\mathrm{Mn}}^{\mathrm{a}} \\
\left(\mathrm{g} \cdot \mathrm{mol}^{-1}\right)\end{array}$ & $\begin{array}{c}\overline{\mathrm{Mw}^{\mathrm{a}}} \\
\left(\mathrm{g} \cdot \mathrm{mol}^{-1}\right)\end{array}$ & $\begin{array}{c}\mathrm{Gd}^{\mathrm{b}} \\
(\%)\end{array}$ & $\begin{array}{c}r_{1}{ }^{\mathrm{c}} \\
\left(\mathrm{mM}^{-1} \mathrm{Gd} \mathrm{s}^{-1}\right)\end{array}$ & $\begin{array}{c}\mathrm{Tb}^{\mathrm{b}} \\
(\%)\end{array}$ & $\begin{array}{c}\text { Fucoidan } \\
\mathrm{mol} / \mathrm{mol} \\
\text { of } \mathrm{P717}\end{array}$ \\
\hline LP717 & 29,000 & 49,000 & $\mathrm{na}$ & - & $\mathrm{na}$ & $\mathrm{na}$ \\
P717Gd & 28,000 & 47,000 & 9.8 & $\mathrm{nd}$ & $\mathrm{na}$ & $\mathrm{na}$ \\
P717Tb & 29,000 & 47,000 & $\mathrm{na}$ & - & 9.7 & na \\
P717Gd-F & 35,000 & 59,000 & 7.3 & 12.3 & - & 1.1 \\
P717Tb-F & 35,000 & 58,000 & - & - & 7.7 & 0.9 \\
\hline
\end{tabular}

aDetermined by SEC-MALLS $( \pm 1000)$.

${ }^{\mathrm{b}}$ Determined by ICP-AES, (weight Gd/weight polymers), SD $=5 \%$.

${ }^{\mathrm{C}}$ Relaxivity values in PBS at $7 \mathrm{~T}, \mathrm{SD}=6 \%$.

${ }^{d}(\mathrm{~mol} / \mathrm{mol})$ determined by colorimetry, SD $=5 \%$.

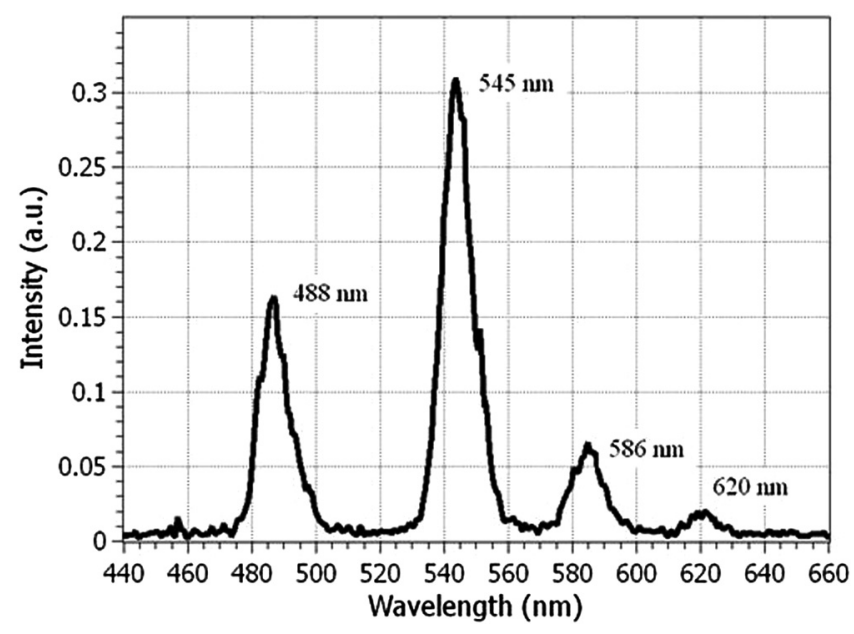

Fig. 3 Spectrum of terbium ions in the P717Tb/PBS solution $\left(\left[\mathrm{Tb}^{3+}\right]=4.3 \mathrm{mM}\right)$. 
(a)

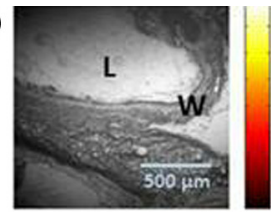

(b)

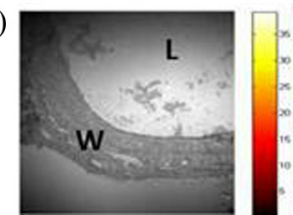

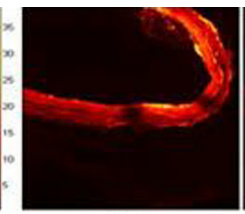

$30 \mathrm{~s}$

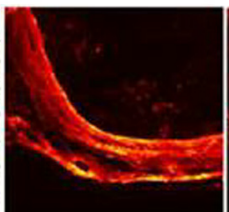

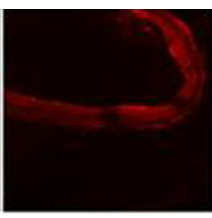

$60 s$

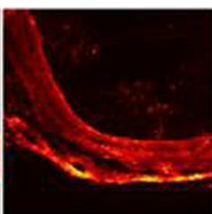

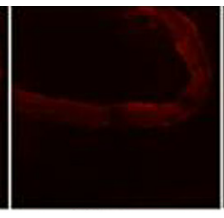

$90 \mathrm{~s}$

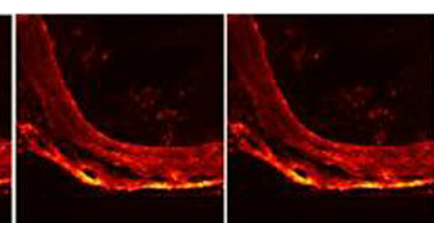

Fig. 4 White field and fluorescent images of healthy artery specimens incubated in PBS (a) without and (b) with P717Tb $([\mathrm{P} 717 \mathrm{~Tb}]=10 \mathrm{mg} / \mathrm{mL})$. The laser is turned on $20 \mathrm{~s}$ after starting the acquisition of images. The peak intensity of laser is about $1500 \mathrm{~W} / \mathrm{cm}^{2}$ to accelerate the photobleaching effect (L, lumen; W, artery wall).

groups and the amounts of $\mathrm{Gd}$ and $\mathrm{Tb}$, a molecular weight increase of about $10 \%$ could be expected instead of the slight decrease (i.e., about $54,000 \mathrm{~g} / \mathrm{mol}$ versus $47,000 \mathrm{~g} / \mathrm{mol}$ to be compared with $49,000 \mathrm{~g} / \mathrm{mol})$. Although we have no explanation, the MWs of both compounds were identical, and the differences with LP717 were not considered drawbacks for the rest of the study. Conversely, the MW increase after aminated fucoidan linking was in accordance with the attachment of one molecule of fucoidan per molecule of P717. Note that each molecule of P717 bears about 20 carboxymethyl groups, but we did not succeed in linking more than one fucoidan per P717. These are average values, and it cannot be excluded that some P717 molecules bear more than one fucoidan while others bear no fucoidan. Eventually, only P717Gd-F and P717Tb-F would interact with $\mathrm{P}$-selectin overexpressed by activated platelets within the thrombus.

The emission spectrum recorded from a solution of $\mathrm{P} 717 \mathrm{~Tb}$ in PBS with a concentration equal to $10 \mathrm{mg} / \mathrm{ml}$ (the molar concentration of terbium ions was $4.6 \times 10^{-3} \mathrm{~mol} / \mathrm{l}$ ) is shown in Fig. 3. We note the four peaks characteristic of terbium ions: $488,545,586$, and $620 \mathrm{~nm}$. It is similar to the spectra published elsewhere. ${ }^{34,43}$ We measured a fluorescence lifetime of terbium complex in PBS of $1911 \pm 8 \mu \mathrm{s}$, consistent with the values published in the literature. ${ }^{43,44}$

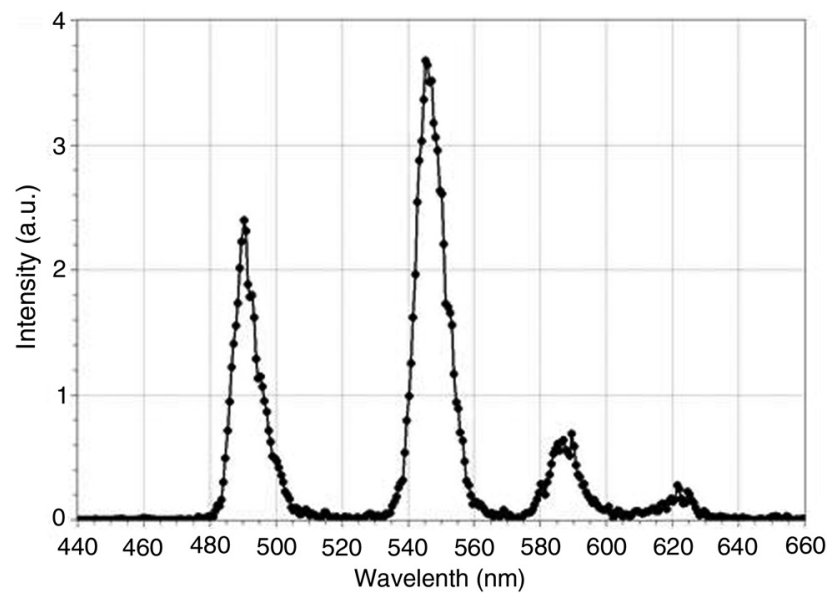

Fig. 5 Emission spectrum of terbium ions in the artery specimen. Artery has been treated with a solution of P717Tb in PBS at the concentration of $10 \mathrm{mg} / \mathrm{ml}$ for $15 \mathrm{~min}$. The samples were carefully rinsed with PBS to remove the excess of P717Tb.

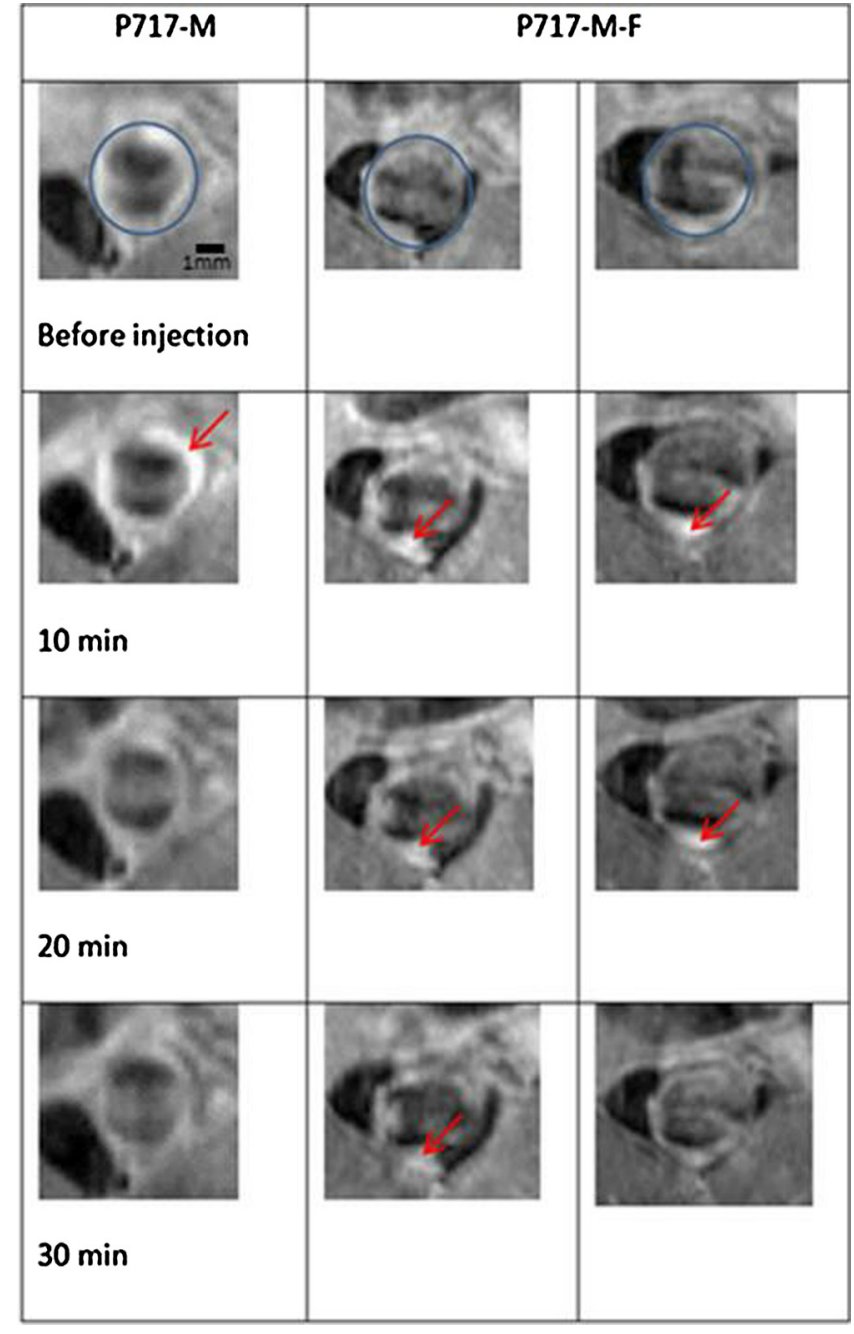

Fig. $6 \mathrm{MR}$ images of pathological arteries after injection into the vein of the tail of rats a mixture of $P 717 \mathrm{M}$, with and without fucoidan $(\mathrm{P} 717 \mathrm{M}-\mathrm{F})(\mathrm{M}=\mathrm{Tb}, \mathrm{Gd} ; 50 / 50 \mathrm{w} / \mathrm{w}$, concentration of each agent $=$ $1 \mathrm{mg} / \mathrm{ml}$ ) to enable them with the capacity to bind to the pathological area in vivo. Red Arrows depict the pathological artery wall areas. Animals were scanned before and after (10, 20, and $30 \mathrm{~min})$ injection of the contrast agent. There was no significant evolution of the MR signal between 30 to $90 \mathrm{~min}$ (data not shown). The scale bar is given on the upper left image. 


\subsection{MR and Optical Imaging}

For this study, we used an abdominal aortic aneurysm (AAA) rat model of atherothrombosis. ${ }^{40}$ The specificity was obtained through the interaction of fucoidan with P-selectin, which is massively expressed by activated platelets. ${ }^{45}$ The thrombus via its associated biological activities including its renewal has been suggested as a driving force for intraplaque hemorrhages in occlusive atherothrombotic disease ${ }^{46}$ as is the case for AAA evolution. ${ }^{13}$ Several authors have visualized thrombus activities using ${ }^{99 \mathrm{~m}} \mathrm{Tc}$ annexin $\mathrm{V}$ in scintigraphy ${ }^{47}$ or iron oxide particle trapping by phagocytes in MRI, ${ }^{48}$ in animal and human AAA. Moreover biological activities detrimental to the arterial wall predominate in the thrombus both in the rat and in humans. ${ }^{49}$ The aneurysmal thrombus is very active, meaning that fresh activated platelets continuously participate in its renewal. Using the rat animal model of elastase-induced abdominal aneurysm, we were previously able to depict with a preclinical MRI a fucoidan-coated USPIO thrombi attached to the aortic wall, with sizes of about $130 \mu \mathrm{m} .{ }^{38}$ However, the contrast agent could not be easily localized on histological slices. With fucoidanlinked $\mathrm{Tb} / \mathrm{Gd}$ complexes, we expected to image the uptake of the contrast agent into the aneurysmal thrombus with MRI as was the case with fucoidan-coated USPIO $^{38}$ and to localize it on histological slices of pathological tissues by optical imaging.

\subsubsection{Healthy arteries imaging}

Fluorescence images of healthy rat artery specimen were compared after incubation in PBS in the absence and presence of P717Tb (Fig. 4). Without the terbium complex, we observed long lifetime emission from the media layer of the artery wall. We should note that this emission only appeared on the media layer of the artery wall. This emission was quickly photobleached, and there was a significant decrease of the intensity. For the specimen treated by the P717Tb $(10 \mathrm{mg} / \mathrm{ml})$, the emission was observed in both media and adventitia layers of the artery wall. The emission spectrum recorded after $140 \mathrm{~s}$ with the
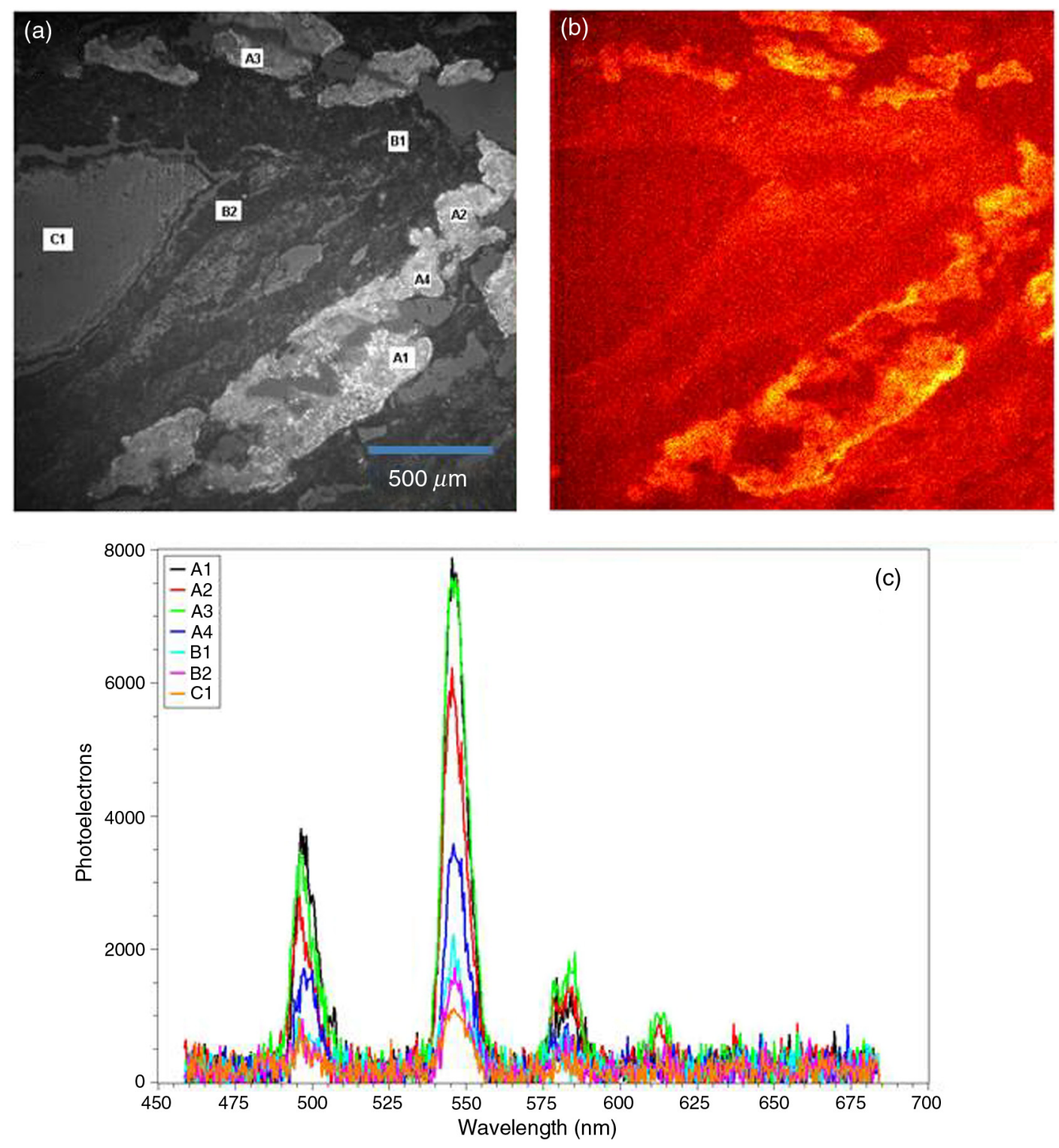

Fig. 7 (a) White light and (b) fluorescence images of a pathological artery slice. (c) Fluorescence spectra at different positions in artery slice (a). The white labels on (a) corresponds to areas where spectra have been collected. 
on-line spectrometer (Fig. 5) evidenced $\mathrm{Tb}^{3+}$ ions as the source of this emission. The long lifetime emission from the media layer of the artery gave a strong noise. Fortunately, it was photobleached quickly while the emission of $\mathrm{Tb}^{3+}$ ions remained stable (data not shown). The bleaching happened after $30 \mathrm{~s}$, which was corresponding to 210 excitation pulses.

\subsubsection{Pathological arteries imaging}

Experiments have been performed with 50/50 (Tb/Gd-w/w) mixtures of P717M and P717M-F with a concentration of $1 \mathrm{mg} / \mathrm{ml}$ for each species before injection in rats. MR phantoms of P717Gd-F solutions in PBS have been imaged to determine the longitudinal relaxivity $r_{1}$ with 7T MRI (see Table 1). The value $12.3 \mathrm{mM}^{-1} \mathrm{Gd} \mathrm{s}^{-1}$ is in accordance with the $r_{1}$ values for P717Gd in the previous studies (7 to $14 \mathrm{mM}^{-1} \mathrm{Gd} \mathrm{s}^{-1}$ ), showing that the coupling with about one molecule of a low molecular weight fucoidan did not significantly modify the magnetic behavior of the contrast agent. In vivo MR images of pathological arteries are shown in Fig. 6. The increase of the Gd concentrations within tissues corresponds to an increase of the bright signal. Two aneurysmal areas have been imaged with the $\mathrm{Tb} / \mathrm{Gd}(50 / 50-\mathrm{w} / \mathrm{w})$ mixture of P717M-F. Increases of the signal within the arterial wall were observed up to $30 \mathrm{~min}$ after injection with P717M-F but not after 10 min with mixtures without fucoidan.

After the euthanization of the animals, the arteries were harvested and slices ( $20 \mu \mathrm{m}$ width) of the pathological section were observed with the fluorescence time-gated microscope. Figure 7 presents results obtained from an animal treated with P717M-F.

The pathological regions appeared brighter in the white light image [Fig. 7(a)] and were clearly correlated to an increase of the fluorescence signal [Fig. 7(b)]. Spectra [Fig. 7(c)] taken at different points of the tissue [indicated by a letter and a digit in Fig. 7(a)] showed the existence of terbium ions in that artery slice. The brighter was the observed region, the stronger were the terbium emission peaks, as expected.

The fluorescence signal recorded on tissue excised from the injected living animal was much lower than the signal recorded from tissue incubated in P717Tb/PBS (Fig. 4). This effect cannot be attributed to the photobleaching by radical species from tissues (ROS) since lanthanide(III) ions are admitted as good quenchers of the triplet state. ${ }^{26}$ Nevertheless, we still got a reasonable SNR due to the high sensitivity of the setup.

This study shows that the P717Tb-F macromolecule can bind to and diffuse into the diseased artery wall and that the autofluorescence intensity collected with our time resolved procedure was negligible compared with the signal emitted by the macromolecular biospecific agent.

\section{Conclusion}

We built a time-resolved fluorescence microscope system for optical imaging of a bimodal mixture of a biospecific contrast agent on healthy and pathological rat arteries: this system was able to locate a fluorescent agent chemically similar to the MR contrast agent used for the detection of an aneurysmal thrombus. For the optical probe, we substituted the magnetic Gd ions with luminescent terbium ions. Fluorescence allowed optical analysis of the interaction between the tissue and the agent. Optical microscopy has a very high intrinsic resolution; therefore, we expected to learn useful information about this interaction. A good SNR was obtained by time-resolved fluorescence imaging, where autofluorescence is fast compared with the terbium emission. We could positively identify terbium spectrum by evaluating the fluorescence spectrum while taking images, which ruled out false positives. In conclusion, we have shown that the P717Tb linked to fucoidan could diffuse into the artery wall and that the autofluorescence intensity collected with our time resolved procedure was negligible compared with the signal emitted with the contrast agent. This is a powerful tool for investigating the interaction of the corresponding MR contrast agents with tissue.

\section{Disclosures}

No conflicts of interest, financial or otherwise, are declared by the authors.

\section{Acknowledgments}

This study was supported by Inserm, University Paris 13, and University Sorbonne Paris Cité. Nguyễn Trọng Nghĩa was awarded a 3 year fellowship from the University Sorbonne Paris Cité. Animal experiments were performed with the help of Ms Liliane Louedec and the pathology department of X. Bichat hospital. The authors also thank Guerbet for the supply of LP717 and Professor Luc Picton from the University of Rouen for his help in physicochemical characterizations.

\section{References}

1. J. A. Finegold, P. Asaria, and D. P. Francis, "Mortality from ischaemic heart disease by country, region, and age: statistics from World Health Organisation and United Nations," Int. J. Cardiol. 168, 934-945 (2013).

2. A. J. Lusis, "Atherosclerosis," Nature 407(6801), 233-241 (2000).

3. D. A. Chistiakov, A. N. Orekhov, and Y. V. Bobryshev, "Contribution of neovascularization and intraplaque haemorrhage to atherosclerotic plaque progression and instability," Acta Physiol. 213(3), 539-553 (2015).

4. Y. Xing et al., "Radiolabeled nanoparticles for multimodality tumor imaging," Theranostics 4(3), 290-306 (2014).

5. M. R. Dweck et al., "Imaging of coronary atherosclerosis-evolution towards new treatment strategies," Nat. Rev. Cardiol. 13(9), 533-548 (2016).

6. T. F. Massoud and S. S. Gambhir, "Molecular imaging in living subjects: seeing fundamental biological processes in a new light," Genes Dev. 17(5), 545-580 (2003).

7. K. Douma et al., "Nanoparticles for optical molecular imaging of atherosclerosis," Small 5(5), 544-557 (2009).

8. J. C. Jung and M. J. Schnitzer, "Multiphoton endoscopy," Opt. Lett. 28(11), 902-904 (2003).

9. J. G. Fujimoto, "Optical coherence tomography for ultrahigh resolution in vivo imaging," Nat. Biotechnol. 21(11), 1361-1367 (2003).

10. S. Sethuraman et al., "Intravascular photoacoustic imaging using an IVUS imaging catheter," IEEE Trans. Ultrason., Ferroelect., Freq. Control 54(5), 978-986 (2007).

11. W. Kerwin et al., "Quantitative magnetic resonance imaging analysis of neovasculature volume in carotid atherosclerotic plaque," Circulation 107(6), 851-856 (2003).

12. N. Takaya et al., "Presence of intraplaque hemorrhage stimulates progression of carotid atherosclerotic plaques-a high-resolution magnetic resonance Imaging study," Circulation 111(21), 2768-2775 (2005).

13. J. B. Michel et al., "Intraplaque haemorrhages as the trigger of plaque vulnerability," Eur. Heart J. 32(16), 1977-1985 (2011).

14. G. C. Makris et al., "Advances in MRI for the evaluation of carotid atherosclerosis," BJR 88(1052), 1-11 (2015).

15. B. Hogers et al., "Magnetic resonance microscopy at 17.6-tesla on chicken embryos in vitro," J. Magn. Reson. Imaging 14(1), 83-86 (2001).

16. K. H. Herrmann et al., "Possibilities and limitations for high resolution small animal MRI on a clinical whole-body 3T scanner," Magn. Reson. Mater. Phys., Biol. Med. 25(3), 233-244 (2012). 
17. E. Kahn et al., "Analysis of the distribution of MRI contrast agents in the livers of small animals by means of complementary microscopies," Cytometry 51A(2), 97-106 (2003).

18. C. Bremer, V. Ntziachristos, and R. Weissleder, "Optical-based molecular imaging: contrast agents and potential medical applications," Eur. Radiol 13(2), 231-243 (2003).

19. I. Hemmila et al., "Europium as a label in time-resolved immunofluorometric assays," Anal. Biochem. 137(2), 335-343 (1984).

20. W. L. Scaff, Jr., D. L. Dyer, and K. Mori, "Fluorescent europium chelate stain,” J. Bacteriol. 98(1), 246-248 (1969).

21. K. Hanaoka, "Development of responsive lanthanide-based magnetic resonance imaging and luminescent probes for biological applications," Chem. Pharm. Bull. 58(10), 1283-1294 (2010).

22. I. Hemmila and S. Webb, "Time-resolved fluorometry: an overview of the labels and core technologies for drug screening applications," Drug Discovery Today 2(9), 373-381 (1997).

23. M. Abdesselem et al., "Multifunctional rare-earth vanadate nanoparticles: luminescent labels, oxidant sensors, and MRI contrast agents," ACS Nano 8(11), 11126-11137 (2014).

24. J. Bünzli, "Lanthanide luminescent bioprobes (LLBs)," Chem. Lett. 38(2), 104-109 (2009).

25. J. Yuan and G. Wang, "Lanthanide complex-based fluorescence label for time-resolved fluorescence bioassay," J. Fluoresc. 15(4), 559568 (2005).

26. J. C. G. Bunzli, "Lanthanide luminescence for biomedical analyses and imaging," Chem. Rev. 110(5), 2729-2755 (2010).

27. J. P. Leonard et al., "Photochemistry and photophysics of coordination compounds: lanthanides," in Photochemistry and Photophysics of Coordination Compounds II, Vol. 281, Topics in Current Chemistry, V. Balzani and S. Campagna, Eds., pp. 1-43, Springer, Berlin Heidelberg (2007)

28. S. Pandya, J. H. Yu, and D. Parker, "Engineering emissive europium and terbium complexes for molecular imaging and sensing," Dalton Trans. 23, 2757-2766 (2006).

29. C. Corot et al., "Physical, chemical and biological evaluations of CMDA2-Gd-DOTA-A new paramagnetic dextran polymer," Acta Radiol. 38, 91-99 (1997).

30. E. P. Canet et al., "Kinetic characterization of CMD-A2-Gd-DOTA as an intravascular contrast agent for myocardial perfusion measurement with MRI," Magn. Reson. Med. 43(3), 403-409 (2000).

31. F. Chaubet et al., "A new macromolecular paramagnetic MR contrast agent binds to activated human platelets," Contrast Media Mol. Imaging 2(4), 178-188 (2007).

32. H. Alsaid et al., "Biomimetic MRI contrast agent for imaging of inflammation in atherosclerotic plaque of ApoE(-/-) mice a pilot study," Invest. Radiol. 44(3), 151-158 (2009).

33. A. Beilvert et al., "Synthesis and evaluation of a tri-tyrosine decorated dextran MR contrast agent for vulnerable plaque detection," Chem. Commun. 47(19), 5506-5508 (2011).
34. M. Elbanowski, S. Lis, and J. Konarski, "Quantum efficiency of the luminescence of $\mathrm{Eu}(\mathrm{III}), \mathrm{Tb}(\mathrm{III})$ and $\mathrm{Dy}(\mathrm{III})$ in aqueous solutions," Monatsh. Chem. 120(8-9), 699-703 (1989).

35. J. J. Freeman, D. E. Lawson, and G. A. Crosby, "Effect of deuterium on luminescence decay times of solvated rare earth chlorides," J. Mol. Spectrosc. 13(4), 399-406 (1964).

36. J. L. Kropp and M. W. Windsor, "Luminescence and energy transfer in solutions of rare-earth complexes. I. Enhancement of fluorescence by deuterium substitution," J. Chem. Phys. 42(5), 1599-1608 (1965).

37. L. Bachelet-Violette et al., "Strong and specific interaction of ultra small superparamagnetic iron oxide nanoparticles and human activated platelets mediated by fucoidan coating," RSC Adv. 4(10), 4864 (2014).

38. M. Suzuki et al., "Ultrasmall superparamagnetic iron oxide nanoparticles coated with fucoidan for molecular MRI of intraluminal thrombus," Nanomedicine 10(1), 73-87 (2015).

39. M. Roth, "Fluorescence reaction for amino acids," Anal. Chem. 43(7), 880-882 (1971).

40. S. Anidjar et al., "Elastase-induced experimental aneurysms in rats," Circulation 82(3), 973-981 (1990).

41. F. Hyafil et al., "Ferumoxtran-10-enhanced MRI of the hypercholesterolemic rabbit aorta-relationship between signal loss and macrophage infiltration," Arterioscler. Thromb. Vasc. Biol. 26(1), 176-181 (2006).

42. R. M. Botnar et al., "In vivo magnetic resonance imaging of coronary thrombosis using a fibrin-binding molecular magnetic resonance contrast agent," Circulation 110(11), 1463-1466 (2004).

43. P. R. Selvin and J. E. Hearst, "Luminescence energy-transfer using a terbium chelate-improvements on fluorescence energy-transfer," Proc. Natl. Acad. Sci. U. S. A. 91(21), 10024-10028 (1994).

44. M. Xiao and P. R. Selvin, "Quantum yields of luminescent lanthanide chelates and far-red dyes measured by resonance energy transfer," J. Am. Chem. Soc. 123(29), 7067-7073 (2001).

45. S. Yokoyama et al., "Platelet P-selectin plays an important role in arterial thrombogenesis by forming large stable platelet-leukocyte aggregates," J. Am. Coll. Cardiol. 45(8), 1280-1286 (2005).

46. J. B. Michel et al., "Novel aspects of the pathogenesis of aneurysms of the abdominal aorta in humans," Cardiovasc. Res. 90(1), 18-27 (2011).

47. L. Sarda-Mantel et al., "Tc-99m-annexin-V functional imaging of luminal thrombus activity in abdominal aortic aneurysms," Arterioscler. Thromb. Vasc. Biol. 26(9), 2153-2159 (2006).

48. A. Nchimi et al., "MR imaging of iron phagocytosis in intraluminal thrombi of abdominal aortic aneurysms in humans," Radiology 254(3), 973-981 (2010).

49. M. Coutard et al., "Thrombus versus wall biological activities in experimental aortic aneurysms," J. Vasc. Res. 47(4), 355-366 (2010).

Biographies for the authors are not available. 